\title{
Current-voltage and kinetic energy flux relations for relativistic field-aligned acceleration of auroral electrons
}

\author{
S. W. H. Cowley \\ Department of Physics \& Astronomy, University of Leicester, Leicester LE1 7RH, UK
}

Received: 24 September 2005 - Accepted: 16 December 2005 - Published: 7 March 2006

\begin{abstract}
Recent spectroscopic observations of Jupiter's "main oval" auroras indicate that the primary auroral electron beam is routinely accelerated to energies of $\sim 100 \mathrm{keV}$, and sometimes to several hundred $\mathrm{keV}$, thus approaching the relativistic regime. This suggests the need to re-examine the classic non-relativistic theory of auroral electron acceleration by field-aligned electric fields first derived by Knight (1973), and to extend it to cover relativistic situations. In this paper we examine this problem for the case in which the source population is an isotropic Maxwellian, as also assumed by Knight, and derive exact analytic expressions for the fieldaligned current density (number flux) and kinetic energy flux of the accelerated population, for arbitrary initial electron temperature, acceleration potential, and field strength beneath the acceleration region. We examine the limiting behaviours of these expressions, their regimes of validity, and their implications for auroral acceleration in planetary magnetospheres (and like astrophysical systems). In particular, we show that for relativistic accelerating potentials, the current density increases as the square of the minimum potential, rather than linearly as in the non-relativistic regime, while the kinetic energy flux then increases as the cube of the potential, rather than as the square.
\end{abstract}

Keywords. Ionosphere (Particle acceleration) - Magnetospheric physics (Auroral phenomena; Magnetosphereionosphere interactions; Current systems)

\section{Introduction}

The exchange of momentum between a magnetised conducting body and its outer plasma envelope via the magnetic field requires the establishment of large-scale electric current systems flowing between them. Stresses are exerted by the cross-field currents flowing in the central body and in the magnetospheric plasma, while the current circuit is closed by field-aligned currents flowing between these re-

Correspondence to: S. W. H. Cowley

(swhc1@ion.le.ac.uk) gions (see e.g. the review by Cowley, 2000, and references therein). The upward-directed field-aligned currents flowing away from the body are generally dominantly carried by precipitating hot magnetospheric electrons, rather than by upflowing cold ionospheric ions. However, for a near-isotropic magnetospheric electron population, the current density that can be carried to the ionosphere by these particles is limited to $j_{\max } \approx e N v_{t h}$, where $e$ is the electron charge, $N$ is the electron number density, constant along a field line for an isotropic population, and $v_{t h}$ is the electron thermal speed. In the terrestrial magnetosphere, for example, the limiting current density is $\sim 0.1 \mu \mathrm{A} \mathrm{m}^{-2}$ for a typical outer plasma sheet electron distribution with a density of $\sim 0.1 \mathrm{~cm}^{-3}$ and a temperature of $\sim 1 \mathrm{keV}$, whilst the current densities implied by the magnetic field perturbations observed in the auroral ionosphere are often more than an order of magnitude larger (e.g. Paschmann et al., 2002, and references therein). When the required current density exceeds the above maximum, an electrostatic potential must develop along the magnetic field lines which accelerates the magnetospheric electrons towards the ionosphere, thereby increasing the current.

The relationship that exists between the field-aligned current carried by precipitating electrons and the field-aligned potential was first studied theoretically by Knight (1973), who showed that under certain simplifying assumptions the minimum electrostatic potential energy required to drive a current which is a factor $F$ times the above maximum, is roughly a factor $F$ times the electron thermal energy. Knight (1973) thus found an approximately linear dependence of the current density on the accelerating potential. Subsequently, Lundin and Sandahl (1978) also showed that the precipitating energy flux of the accelerated electron population is then amplified by roughly a factor of $F^{2}$ compared with the unaccelerated population, thus leading to major enhancements in electron energy input to the ionosphere, and consequently also to auroral output. Knight's (1973) theory has subsequently been extensively applied to terrestrial studies of "inverted- $\mathrm{V}$ " electron precipitation and substorm-related auroras (e.g. Antonova and Tverskoy, 1975; Lyons, 1980, 1981; Bosqued et al., 1986; Shiokawa et 
al., 1990; Lu et al., 1991; Olsson et al., 1996, 1998; Schriver et al., 2003), as well as to auroras in the dayside cusp (Korth et al., 2004), and in the polar cap (Carlson and Cowley, 2005). Field-aligned potentials of $\sim 1-$ $10 \mathrm{kV}$ are typically required in upward-directed auroral current regions in the terrestrial case, whose effects are routinely observed in the particle distributions on auroral field lines (e.g. Paschmann et al., 2002). Knight's (1973) theoretical formulation has also subsequently been extended beyond the isotropic Maxwellian velocity distributions he considered, to also include bi-Maxwellian and kappa distributions (Fridman and Lemaire, 1980; Pierrard, 1996; Janhunen and Olsson, 1998; Dors and Kletzing, 1999). However, the results obtained do not differ qualitatively from those found previously by Knight (1973).

The most recent application of Knight's (1973) theory has been to the current systems and auroras of the planets Jupiter and Saturn (Cowley and Bunce, 2001; Cowley et al., 2004, 2005; Nichols and Cowley, 2004). In the case of Jupiter's magnetosphere (but not Saturn's), the principal current system is that associated with the transfer of planetary angular momentum to the magnetospheric plasma, rather than with the solar wind interaction as at Earth. This current system is associated with a narrow and intense ring of upward-directed field-aligned current surrounding the magnetic pole, whose current density at ionospheric heights, $\sim 0.1$ to $\sim 0.5 \mu \mathrm{A} \mathrm{m}^{-2}$, considerably exceeds the maximum that can be carried by unaccelerated magnetospheric electrons, $\sim 0.01 \mu \mathrm{A} \mathrm{m}^{-2}$. Consequently, the electrons must be strongly accelerated along the field lines from a few $\mathrm{keV}$ to energies of $\sim 100 \mathrm{keV}$ and above, as indicated both by theory (e.g. Cowley et al., 2003), and by observations of the spectra of the resulting intense "main oval" UV auroras (Gustin et al., 2004). Indeed, on some occasions, the accelerated electron energies are deduced by these means to reach to at least a few hundred $\mathrm{keV}$, thus approaching the relativistic regime.

These observations suggest the need to extend Knight's (1973) theory to encompass relativistic situations, in which the plasma electrons are either very energetic initially, or become so due to the presence of field-aligned potentials which are comparable with or exceed the electron rest energy $(\sim 511 \mathrm{keV})$. Such field-aligned potentials are not implausible in Jupiter's magnetosphere, for example, since the total cross-field potential across the outer and middle magnetosphere region is of order $\sim 10 \mathrm{MV}$ (e.g. Nichols and Cowley, 2005; Cowley et al., 2005). In addition, a number of applications to astrophysical systems have also been proposed which clearly require a relativistic treatment (e.g. Ergun et al., 2000; Haerendel, 2001; Begelman et al., 2005). In this paper we thus present a relativistic formulation of Knight's (1973) kinetic theory, and derive exact analytic expressions for the field-aligned current density (number flux) and kinetic energy flux of the accelerated particles, for arbitrary initial temperature, accelerating potential, and magnetic field strength beneath the voltage drop.

\section{Theory}

We consider a situation in which magnetospheric electrons move along magnetic field lines toward a magnetised planet, such that the field strength at the particle is steadily increasing. At some point where the field strength is $B_{o}$ the electrons encounter a region of field-aligned electric field which is assumed compact along the field lines, such that they are accelerated along the field towards the planet. Beneath the voltage drop the field strength continues to grow such that the accelerated electron population becomes modified by magnetic mirroring. Here we wish to calculate the current density and kinetic energy flux of the accelerated electrons, in terms of the unaccelerated electron parameters, for arbitrary accelerating potential and magnetic field strength relative to $B_{o}$. The calculation consists of three steps. We first consider the motion of single particles in the above fields, to determine the relationship between the momentum components before and after acceleration. Second, we use this information to determine the properties of the accelerated particle distribution function in terms of the unaccelerated distribution, using $\mathrm{Li}$ ouville's theorem. Third, we then obtain the current density and kinetic energy flux by integration of the appropriate moments over the distribution function. Here we now consider each of these steps in turn.

\subsection{Single particle motion}

The motion of particles in the above fields is governed by conservation of two invariant quantities. The first is the total energy $\mathcal{E}+q \phi$, where $\phi$ is the electrostatic potential, $q$ is the particle charge, and $\mathcal{E}$ is the relativistic energy of the particle given by

$\mathcal{E}=\sqrt{p^{2} c^{2}+m^{2} c^{4}}$,

where $p$ is the magnitude of the relativistic momentum of the particle, $m$ is its rest mass, and $c$ the speed of light. The total energy is an exact invariant of the motion for static fields, as considered here, as is readily shown by direct integration of the relativistic equation of motion (e.g. Clemmow and Dougherty, 1969). The second is the magnetic moment invariant associated with the gyration of particles around the field lines, which can be written as $p_{\perp}^{2} / B$, where $p_{\perp}$ is the momentum component perpendicular to the magnetic field, and $B$ is the magnetic field strength. This is an adiabatic invariant which is preserved provided that the scale length of field variation is large compared with the particle gyroradius, as will be assumed here. We also define the pitch angle of the particle $\alpha$ to be the angle between the momentum vector and the magnetic field, such that the perpendicular component of the momentum can be written as $p_{\perp}=p \sin \alpha$, while the parallel component is $p_{\|}=p \cos \alpha$.

Let us consider an electron whose energy and pitch angle just before impinging on the acceleration region at field strength $B_{o}$ are $\mathcal{E}_{o}$ (corresponding to momentum $p_{o}$ through Eq. 1) and $\alpha_{o}$, respectively. After passing through the acceleration region and reaching a point on the field line where 
the field strength is $B$, conservation of total energy and the first adiabatic invariant then show that the particle energy $\mathcal{E}$ (corresponding to momentum $p$ ) and pitch angle $\alpha$ are determined by

$\mathcal{E}=\mathcal{E}_{o}+e \Phi$

or equivalently

$\sqrt{p^{2} c^{2}+m^{2} c^{4}}=\sqrt{p_{o}^{2} c^{2}+m^{2} c^{4}}+e \Phi$,

and

$$
\frac{p^{2} \sin ^{2} \alpha}{B}=\frac{p_{o}^{2} \sin ^{2} \alpha_{o}}{B_{o}},
$$

where $\Phi$ is the total potential drop along the field lines, and $e$ is the electron charge (taken to be a positive quantity). Equation (2) determines the relationship between the energies $\mathcal{E}_{o}$ and $\mathcal{E}$ above and below the voltage drop (or equivalently between the magnitudes of the momenta $p_{o}$ and $p$ ), while Eq. (3) then determines the relationship between the pitch angles. Since all the distribution functions we consider are assumed gyrotropic around the field direction, these equations are then sufficient to map the electron distribution function from above to below the acceleration region.

\subsection{Mapping the distribution function}

In general the particle distribution function $f$ is defined such that the number of particles in phase space volume $d^{3} \boldsymbol{r} d^{3} \boldsymbol{p}$ at position vector $\boldsymbol{r}$ and momentum vector $\boldsymbol{p}$ at time $t$ is $f(\boldsymbol{r}, \boldsymbol{p}, t) d^{3} \boldsymbol{r} d^{3} \boldsymbol{p}$. The distribution function is then mapped in phase space using Liouville's theorem, which states that $f$ is constant on a particle trajectory (e.g. Clemmow and Dougherty, 1969). Here we consider a steady state in which the only spatial variation is along the magnetic field lines. The accelerated distribution function $f$ and the unaccelerated distribution function $f_{o}$ are then simply related by

$f(p, \alpha)=f_{o}\left(p_{o}, \alpha_{o}\right)$,

where $p$ and $\alpha$ are related to $p_{o}$ and $\alpha_{o}$ by Eqs. (2b) and (3). Equation (4a) thus directly determines the accelerated distribution function below the voltage drop in terms of the distribution function immediately above it. Here for simplicity we only consider unaccelerated distributions that are isotropic, and hence independent of position along the field line above the voltage drop, in which case we have simply

$$
f(p)=f_{o}\left(p_{o}\right) \quad \text { or equivalently } f(\mathcal{E})=f_{o}\left(\mathcal{E}_{o}\right),
$$

where $p$ and $p_{o}$, or equivalently $\mathcal{E}$ and $\mathcal{E}_{o}$, are related through Eq. (2).

Although any isotropic distribution can be considered in principle, here we also assume for simplicity that the unaccelerated distribution is Maxwellian in form, thus following the formulation employed originally by Knight (1973), and used in most of the applications cited in the introduction. We thus take the unaccelerated population to be given by

$$
f_{o}\left(\mathcal{E}_{o}\right)=f_{o} \exp \left(-\frac{\left(\mathcal{E}_{o}-m c^{2}\right)}{k T}\right) \text {, }
$$

where $f_{o}$ is a constant to be defined (in the following section) in terms of the unaccelerated electron density, $T$ is the electron temperature, and $k$ is Boltzmann's constant. We note that $W_{o}=\mathcal{E}_{o}-m c^{2}$ is the initial particle kinetic energy. From Eqs. (2a) and (4b), the accelerated distribution function is then given simply by

$f(\mathcal{E})=f_{o} \exp \left(-\frac{\left(\mathcal{E}-e \Phi-m c^{2}\right)}{k T}\right)$.

Care must be exercised in interpreting these expressions, however, since in general not all of the momentum space of the accelerated distribution is populated by particles from the unaccelerated distribution to give the values indicated in Eqs. (4) or (6). The populated volume corresponds only to those particles which move along the field line in the appropriate direction in the region above the voltage drop to impinge on the acceleration region from above, the limiting case being that of particles which are locally mirroring at $90^{\circ}$ pitch angle at field strength $B_{o}$. Setting $\alpha_{o}=90^{\circ}$ in Eq. (3) and eliminating $p_{o}$ between Eqs. (2) and (3) then yields the following quadratic expression for the limiting energy $\mathcal{E}_{L}(\alpha)$ below which the accelerated distribution is unpopulated at pitch angle $\alpha$

$$
\begin{aligned}
\mathcal{E}_{L}^{2}(\alpha) & -\frac{2 e \Phi}{\left(1-\frac{B_{o}}{B} \sin ^{2} \alpha\right)} \mathcal{E}_{L}(\alpha) \\
- & m^{2} c^{4}+\frac{(e \Phi)^{2}}{\left(1-\frac{B_{o}}{B} \sin ^{2} \alpha\right)}=0 .
\end{aligned}
$$

The physically acceptable root of this expression which reduces to $\mathcal{E}_{L}(\alpha) \rightarrow m c^{2}$ for all $\alpha$ when $e \Phi \rightarrow 0$ (i.e. the limiting kinetic energy $W_{L}(\alpha)=\mathcal{E}_{L}(\alpha)-m c^{2}$ goes to zero at all pitch angles in this limit as required) is

$$
\mathcal{E}_{L}(\alpha)=\frac{e \Phi}{\left(1-\frac{B_{o}}{B} \sin ^{2} \alpha\right)}+\sqrt{m^{2} c^{4}+\frac{\frac{B_{o}}{B} \sin ^{2} \alpha(e \Phi)^{2}}{\left(1-\frac{B_{o}}{B} \sin ^{2} \alpha\right)^{2}}} .
$$

The limiting energy for particles moving exactly along the field direction with $\alpha=0$ is thus $\mathcal{E}_{L}(0)=e \Phi+m c^{2}$ for all $B$, as expected (i.e. the limiting kinetic energy along the field direction is just the electrostatic potential energy $e \Phi)$. Limiting energy $\mathcal{E}_{L}(\alpha)$ (and kinetic energy $W_{L}(\alpha)$ ) then in general increase monotonically with the pitch angle for given $B$, reaching the value

$\mathcal{E}_{L}\left(\frac{\pi}{2}\right)=\frac{e \Phi}{\left(1-\frac{B_{o}}{B}\right)}+\sqrt{m^{2} c^{4}+\frac{\frac{B_{o}}{B}(e \Phi)^{2}}{\left(1-\frac{B_{o}}{B}\right)^{2}}}$,

at $90^{\circ}$. However, in the limit that $B$ becomes very large compared with $B_{o}$, we then find $\mathcal{E}_{L}(\alpha) \rightarrow e \Phi+m c^{2}$ for all $\alpha$ (i.e. $\left.W_{L}(\alpha) \rightarrow e \Phi\right)$, due to the magnetic mirroring of the accelerated particles beneath the voltage drop. The "hole" in the distribution function at low energies then becomes spherical in momentum space in this limit. 

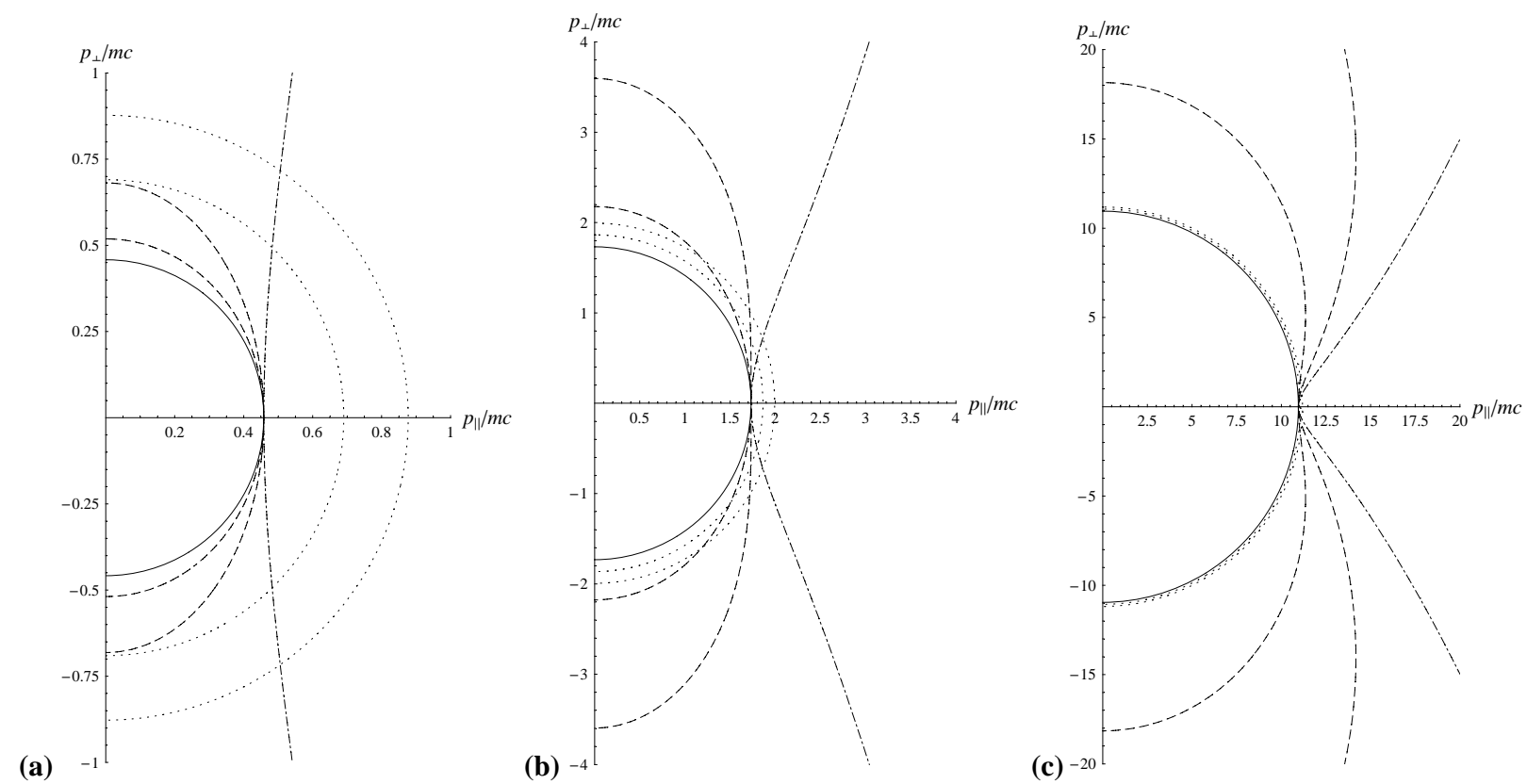

Fig. 1. Cuts through momentum space in the $\left(p_{\perp}, p_{\|}\right)$plane, showing contours of constant distribution function (solid and dotted circles) for an initially Maxwellian distribution with non-relativistic temperature such that $\left(m c^{2} / k T\right)=20$ which is accelerated through field-aligned potentials of $\left(e \Phi / m c^{2}\right)=0.1,1$, and 10 in panels (a), (b), and (c), respectively. The solid circle in each plot represents the inner limit of the distribution (for $\left(B / B_{O}\right) \rightarrow \infty$ ), while the inner and outer dotted circles show the surfaces where the distribution function falls to factors of 0.1 and 0.01 of the value on the solid circle, respectively (given by Eq. 6 combined with Eq. 1). These dotted contours can only just be seen outside the solid circle in case (c). The dot-dashed and dashed lines then show where these distributions are truncated in momentum space for finite $\left(B / B_{O}\right)$ beneath the voltage drop, given by Eq. $(7 \mathrm{~b})$, the full surfaces being obtained by revolution about the $p_{\|}$axis. The dot-dashed line in each plot corresponds to $\left(B / B_{O}\right)=1$ directly beneath the voltage drop, while the two dashed lines correspond to $\left(B / B_{O}\right)=2$ and 5 . The solid line shows the limiting surface for $\left(B / B_{O}\right) \rightarrow \infty$, such that the accelerated distribution then becomes fully isotropic.

We note that the non-relativistic limit of Eq. (7b), in which $\left(e \Phi / m c^{2}\right) \ll 1$ such that the square root on the RHS reduces to $m c^{2}$, is then given by

$W_{L}(\alpha)=\mathcal{E}_{L}(\alpha)-m c^{2} \approx \frac{e \Phi}{\left(1-\frac{B_{o}}{B} \sin ^{2} \alpha\right)}$,

such that the "hole" in the distribution function is then generally an ellipsoid of revolution about the field direction, reducing to a sphere in the limit $\left(B / B_{o}\right) \rightarrow \infty$ as above. In the opposite limit where $\left(B / B_{O}\right) \rightarrow 1$, directly beneath the acceleration region, Eq. (8) becomes $W_{L}(\alpha) \cos ^{2} \alpha \approx e \Phi$, i.e. the surface of the "hole" in the non-relativistic case is simply the plane $v_{\| L}=\sqrt{2 e \Phi / m}$, where $v_{\|}$is the field-aligned component of the particle velocity.

Some examples of the limiting surfaces obtained from Eq. (7b) (and Eq. 1) are illustrated in Fig. 1. Here we show cuts through momentum space, with $p_{\|}$plotted on the horizontal axis and $p_{\perp}$ on the vertical axis, with momentum values being normalised to $m c$. The limiting surfaces are surfaces of revolution about the $p_{\|}$axis, shown by lines in the plane of the cut. In each diagram the dot-dashed line indicates the limiting surface for $\left(B / B_{o}\right)=1$, directly beneath the accelerating voltage, while the solid line (a circle) shows the surface for $\left(B / B_{o}\right)=\infty$. The dashed lines between then show the surfaces for $\left(B / B_{O}\right)=2$ and 5 . (The dotted lines will be discussed below.) The "hole" in the distribution function for a particular value of $\left(B / B_{o}\right)$ then corresponds to the volume bounded by these surfaces, containing the origin of momentum space. In Fig. 1a we show the surfaces for $\left(e \Phi / m c^{2}\right)=0.1$ (i.e. an accelerating potential of $\sim 50 \mathrm{kV}$ for electrons), such that in this case we approach the nonrelativistic regime. It can be seen that the limiting surface for $\left(B_{o} / B\right)=1$ is close to a plane at fixed $p_{\|}$as anticipated above, though the curve is perceptibly convex with respect to the origin. At higher values of $\left(B / B_{o}\right)$ the curves then approach the ellipsoidal forms indicated by Eq. (8), eventually becoming circular as $\left(B / B_{o}\right) \rightarrow \infty$. Figures $1 \mathrm{~b}$ and c correspond to relativistic cases in which $\left(e \Phi / m c^{2}\right)=1$ and 10 , respectively, i.e. to accelerating potentials for electrons of $\sim 500 \mathrm{kV}$ and $\sim 5 \mathrm{MV}$. In these cases the convex nature of the curves for small $\left(B / B_{o}\right)$ and small pitch angle becomes considerably more accentuated, and the curves approach circularity less rapidly with increasing $\left(B / B_{O}\right)$ than in the nonrelativistic case. 
We now consider the accelerated Maxwellian distribution given by Eq. (6) in relation to these surfaces. We note that at zero pitch angle the limiting energy is $\mathcal{E}_{L}(0)=e \Phi+m c^{2}$ as indicated above, equal to $\mathcal{E}_{L}(\alpha)$ for all $\alpha$ when $\left(B / B_{o}\right) \rightarrow \infty$. Substitution into Eq. (6) then shows that the accelerated distribution function has its maximum value at this energy, corresponding to $\left(f / f_{o}\right)=1$. Thus the accelerated distribution function has its maximum value on the surface of the limiting spherical "hole" which is present when $\left(B / B_{o}\right) \rightarrow \infty$, shown by the solid circle in Fig. 1, and falls in value exponentially at larger energies, on scale $k T$. As an illustration, the dotted lines in Fig. 1 show surfaces of constant distribution function (spheres) of the accelerated population for a particular case in which $\left(m c^{2} / k T\right)=20$, corresponding to a non-relativistic unaccelerated electron population of thermal energy $\sim 25 \mathrm{keV}$. The distribution function then has the value $\left(f / f_{o}\right)=1$ on the solid circle as just indicated, but falls to $\left(f / f_{o}\right)=0.1$ and 0.01 , respectively, on the inner and outer dotted lines illustrated in each panel. It can be seen that the accelerated population becomes an increasingly thin shell in momentum space as the accelerating potential is increased. The relationship between these dotted contours and the lines showing the limiting surfaces in these panels then also graphically illustrates how the accelerated population is increasingly focussed along the field direction in momentum space as the accelerating potential increases, and is then spread in pitch angle by the increasing field strength. It is evident, however, that the increase in field strength required to render the distribution function near-isotropic in Fig. 1c is much larger than that required at lower accelerating potential in Fig. 1a.

\subsection{Bulk parameters}

The bulk parameters associated with the unaccelerated and accelerated distributions are determined by obtaining suitable moments of the distribution function. Those of particular interest here are the number density $N$, the field-aligned current density $j_{\|}$(the field-aligned number flux times the charge), and the field-aligned kinetic energy flux $\mathcal{W}_{\|}$. These are given by

$N=\int f d^{3} \boldsymbol{p}$

$j_{\|}=e \int v_{\|} f d^{3} \boldsymbol{p}$

and

$\mathcal{W}_{\|}=\int v_{\|}\left(\mathcal{E}-m c^{2}\right) f d^{3} \boldsymbol{p}$

where $v_{\|}$is the field-aligned particle velocity, and the particle charge has simply been written as $e$. To obtain a suitable relativistic expression for $v_{\|}$, we note that the particle relativistic momentum is $\boldsymbol{p}=\boldsymbol{M v}$, where $\boldsymbol{v}$ is the particle velocity, and $M$ is the relativistic mass (as opposed to the rest mass $m$ employed above). We also have $\mathcal{E}=M c^{2}$, so that in terms of $p$ and $\mathcal{E}$

$v_{\|}=\frac{p c^{2} \cos \alpha}{\mathcal{E}}$.

The volume element in momentum space in spherical polar $(p, \alpha)$ co-ordinates is

$d^{3} \boldsymbol{p}=2 \pi p^{2} \sin \alpha d p d \alpha=\frac{2 \pi p \mathcal{E}}{c^{2}} \sin \alpha d \mathcal{E} d \alpha$,

where we have integrated in azimuth around the field direction assuming a gyrotropic distribution as above, and the second form involving integration over relativistic energy $\mathcal{E}$ rather than momentum $p$ follows from Eq. (1). Substituting Eqs. (10) and (11) into Eq. (9) then yields the general forms

$N=\frac{2 \pi}{c^{3}} \int d \alpha \sin \alpha \int d \mathcal{E} \sqrt{\mathcal{E}^{2}-m^{2} c^{4}} \mathcal{E} f$,

$j_{\|}=\frac{2 \pi e}{c^{2}} \int d \alpha \sin \alpha \cos \alpha \int d \mathcal{E}\left(\mathcal{E}^{2}-m^{2} c^{4}\right) f$,

and

$\mathcal{W}_{\|}=\frac{2 \pi}{c^{2}} \int d \alpha \sin \alpha \cos \alpha \int d \mathcal{E}\left(\mathcal{E}^{2}-m^{2} c^{4}\right)\left(\mathcal{E}-m c^{2}\right) f$

We first apply these expressions to the unaccelerated population whose distribution function is given by Eq. (5). Substituting into Eq. (12a) and integrating over the whole of momentum space (from $m c^{2}$ to infinity in $\mathcal{E}$, and 0 to $\pi$ in $\alpha$ ), then yields the density as

$N_{o}=4 \pi f_{o}(m c)^{3} \frac{\exp (\mu) K_{2}(\mu)}{\mu}$,

where $\mathrm{K}_{2}$ is the modified Bessel function of the second kind of order 2, and for simplicity of notation we have written the dimensionless ratio of the rest energy to the thermal energy as $\mu$, i.e.

$\mu=\frac{m c^{2}}{k T}$.

The constant $f_{o}$ in the distribution function is thus obtained in terms of the density as

$f_{o}=\frac{N_{o}}{4 \pi(m c)^{3}} \frac{\mu}{\exp (\mu) K_{2}(\mu)}$.

The non-relativistic limit is given by putting $\mu \gg 1$, and noting that the asymptotic form for the Bessel function for large $z$ is $e^{z} K_{v}(z) \approx \sqrt{\pi / 2 z}$, we thus recover the non-relativistic result

$f_{o} \approx \frac{N_{o}}{m^{3}}\left(\frac{m}{2 \pi k T}\right)^{3 / 2}$

Substitution of Eq. (5) into Eqs. (12b) and (12c) then yields the field-aligned current density and kinetic energy flux of the unaccelerated distribution for one hemisphere of momentum space only as

$j_{\| o}=\frac{2 \pi e f_{o}(k T)^{3}}{c^{2}}(\mu+1)$, 
and

$\mathcal{W}_{\| o}=\frac{2 \pi f_{o}(k T)^{4}}{c^{2}}(2 \mu+3)$,

where $f_{o}$ is given by Eq. (15). These expressions correspond to the fluxes of particles moving in one direction only along the field, e.g. toward the planet and the acceleration region, such that the integral over $\alpha$ is from 0 to $\pi / 2$ only. We also note that because the unaccelerated population is assumed isotropic, these quantities (and the particle number density) are constant along the field lines above the voltage drop. In the non-relativistic limit, substitution of Eq. (16) into Eq. (17) then yields the familiar expressions (e.g. Cowley et al., 2003)

$j_{\| o} \approx e N_{o}\left(\frac{k T}{2 \pi m}\right)^{1 / 2}$

and

$\mathcal{W}_{\| o} \approx 2 N_{o} k T\left(\frac{k T}{2 \pi m}\right)^{1 / 2}$.

Equation (18a) is essentially just $j_{\|} \approx e N v_{t h}$ as indicated in the introduction.

We now similarly obtain the required expressions for the field-aligned current density and kinetic energy flux of the accelerated population, depending on the initial thermal energy $k T$, the accelerating potential energy $e \Phi$, and the magnetic field ratio $\left(B / B_{o}\right)$. Again for simplicity of notation we write related dimensionless ratios as

$\varphi=\frac{e \Phi}{k T}$

and

$\beta=\frac{B}{B_{o}}$.

We first examine the field-aligned current density, obtained by substituting Eq. (6) into Eq. (12b), and integrating from the limiting energy $\mathcal{E}_{L}(\alpha)$ given by Eq. (7b) to infinity in $\mathcal{E}$, and from 0 to $\pi / 2$ in $\alpha$. Performing the energy integral first yields

$$
\begin{aligned}
\left(\frac{j_{\|}}{j_{\| o}}\right)= & \frac{1}{(\mu+1)} \int_{0}^{\pi / 2} d \alpha \sin \alpha \cos \alpha F\left(\varepsilon_{L}(\alpha)\right) \\
& \times \exp \left(-\left(\varepsilon_{L}(\alpha)-\varphi-\mu\right)\right),
\end{aligned}
$$

where the dimensionless ratio

$\varepsilon_{L}(\alpha)=\frac{\mathcal{E}_{L}(\alpha)}{k T}$,

function $F\left(\varepsilon_{L}(\alpha)\right)$ is given by

$F\left(\varepsilon_{L}(\alpha)\right)=\varepsilon_{L}^{2}(\alpha)+2\left(\varepsilon_{L}(\alpha)+1\right)-\mu^{2}$,

and where we have normalized $j_{||}$to the corresponding current density $j_{\| o}$ of the unaccelerated population given by
Eq. (17a), thus eliminating $f_{o}$ proportional to the unaccelerated density $N_{o}$. The integration over pitch angle can then most easily be accomplished by transforming the integration variable from $\alpha$ to $\varepsilon_{L}(\alpha)$ using Eq. (7a), such that

$\sin \alpha \cos \alpha d \alpha=\varphi \beta \frac{\left(\varepsilon_{L}^{2}-\varphi \varepsilon_{L}+\mu^{2}\right)}{\left(\varepsilon_{L}^{2}-\mu^{2}\right)^{2}} d \varepsilon_{L}$.

Integration then yields the relativistic general result

$\left(\frac{j_{\|}}{j_{\| o}}\right)=\beta\left[1-\frac{\varphi \tilde{F}\left(\varepsilon_{L}\left(\frac{\pi}{2}\right)\right) \exp \left(-\left(\varepsilon_{L}\left(\frac{\pi}{2}\right)-\varphi-\mu\right)\right)}{(\mu+1)\left(\varepsilon_{L}\left(\frac{\pi}{2}\right)^{2}-\mu^{2}\right)}\right]$,

where

$\tilde{F}\left(\varepsilon_{L}\left(\frac{\pi}{2}\right)\right)=\varepsilon_{L}^{2}\left(\frac{\pi}{2}\right)-\varepsilon_{L}\left(\frac{\pi}{2}\right)(\varphi-2)+\left(\mu^{2}-\varphi\right)$,

and $\varepsilon_{L}\left(\frac{\pi}{2}\right)$ is given by Eqs. (7c) and (21b). The nonrelativistic limit is obtained by putting

$\varepsilon_{L}\left(\frac{\pi}{2}\right) \approx \frac{\varphi \beta}{(\beta-1)}+\mu$

from Eq. (8), and retaining only the highest order terms in $\mu$. Equation (23) then reduces to

$$
\left(\frac{j_{\|}}{j_{\| o}}\right) \approx \beta-(\beta-1) \exp \left(-\frac{\varphi}{(\beta-1)}\right),
$$

which is the result derived previously by Knight (1973) and subsequent authors.

The related relativistic expressions for the field-aligned kinetic energy flux of the accelerated electrons, also normalized to the value for the unaccelerated population, obtained from Eqs. (6), (12c), and (17b), are as follows. Integrating first in energy, as above, yields

$$
\begin{aligned}
\left(\frac{\mathcal{W}_{\|}}{\mathcal{W}_{\| o}}\right)= & \frac{1}{(2 \mu+3)} \int_{0}^{\pi / 2} d \alpha \sin \alpha \cos \alpha G\left(\varepsilon_{L}(\alpha)\right) \\
& \times \exp \left(-\left(\varepsilon_{L}(\alpha)-\varphi-\mu\right)\right),
\end{aligned}
$$

where

$$
\begin{aligned}
G\left(\varepsilon_{L}(\alpha)\right)= & \varepsilon_{L}^{3}(\alpha)-\varepsilon_{L}^{2}(\alpha)(\mu-3) \\
& -\left(\varepsilon_{L}(\alpha)+1\right)\left(\mu^{2}+2 \mu-6\right)+\mu^{3} .
\end{aligned}
$$

Transforming the pitch angle integral according to Eq. (22) and integrating, we then find the relativistic general result

$$
\begin{aligned}
\left(\frac{\mathcal{W}_{\|}}{\mathcal{W}_{\| o}}\right) & =\beta\left[1+\frac{\varphi(\mu+1)}{(2 \mu+3)}\right. \\
& \left.-\frac{\varphi \tilde{G}\left(\varepsilon_{L}\left(\frac{\pi}{2}\right)\right) \exp \left(-\left(\varepsilon_{L}\left(\frac{\pi}{2}\right)-\varphi-\mu\right)\right)}{(2 \mu+3)\left(\varepsilon_{L}^{2}\left(\frac{\pi}{2}\right)-\mu^{2}\right)}\right],
\end{aligned}
$$

where

$$
\begin{aligned}
\tilde{G}\left(\varepsilon_{L}\left(\frac{\pi}{2}\right)\right)= & \varepsilon_{L}^{3}\left(\frac{\pi}{2}\right)-\varepsilon_{L}^{2}\left(\frac{\pi}{2}\right)(\mu+\varphi-4) \\
& +\varepsilon_{L}\left(\frac{\pi}{2}\right)(\mu(\mu-2)+\varphi(\mu-3)+6) \\
& +\left(\varphi(\mu-3)-\mu^{2}(\mu-2)\right) .
\end{aligned}
$$


(a)

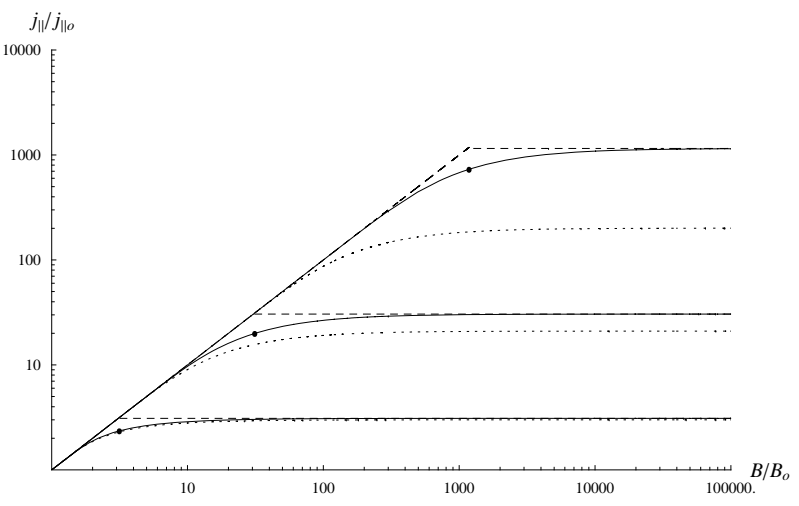

(b)

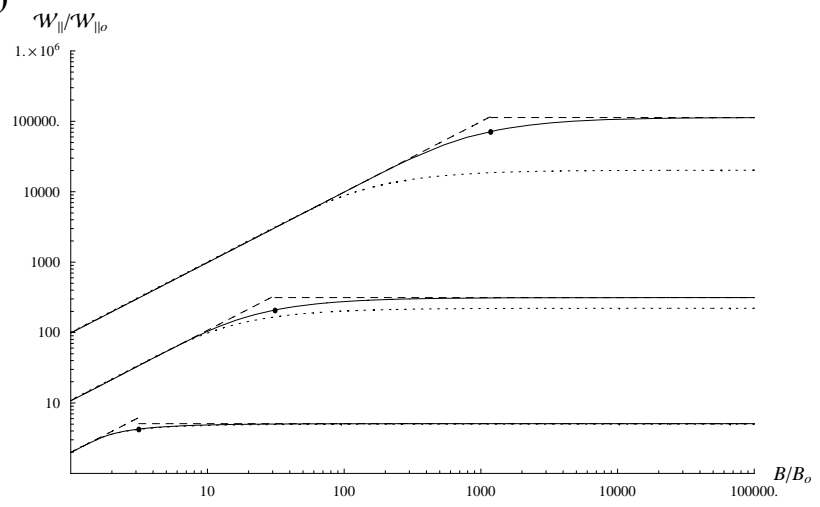

Fig. 2. Plots showing the variation of (a) the normalised field-aligned current density $\left(j_{\|} / j_{\| o}\right)$ (or equivalently the number flux) and (b) the normalised field-aligned kinetic energy flux $\left(\mathcal{W}_{\| \mid} / \mathcal{W}_{\| \mid}\right)$of the accelerated population, versus $\left(B / B_{o}\right)$ beneath the voltage drop, for fixed values of the accelerating potential $\Phi$ and thermal energy $k T$ of the unaccelerated population. The fluxes are normalized in each case to the corresponding values for the unaccelerated population, given by Eq. (17). All curves are for $\left(m c^{2} / k T\right)=20$ (corresponding to $k T \sim 25 \mathrm{keV}$ for electrons), while the lower, middle, and upper curves in each panel are for $(e \Phi / k T)=2,20$, and 200 (corresponding to $e \Phi \sim 50,500$, and $5000 \mathrm{keV}$ for electrons), respectively, such that $\left(e \Phi / m c^{2}\right)=0.1,1$, and 10 . The solid lines in each panel show the relativistic values given by Eq. (23) for the current density and Eq. (26) for the kinetic energy flux, while the dotted lines show the non-relativistic approximations given by Eqs. (24) and (27). The dashed lines show the limiting behaviours for small and large $\left(B / B_{O}\right)$, given by Eqs. (28) and (29) for small $\left(B / B_{O}\right)$, and Eqs. (33) and (34) for large $\left(B / B_{O}\right)$. The transition from one dashed line limit to the other has been taken to occur at $\left(B / B_{O}\right)=\left(B / B_{O}\right)_{\lim A-B}$ given by Eq. (37), at which point a solid dot is shown on the solid lines, marking the transition between these limiting behaviours.

The non-relativistic limit, obtained in the same way as for the current density above, is

$$
\left(\frac{\mathcal{W}_{\|}}{\mathcal{W}_{\| o}}\right) \approx \beta\left(1+\frac{\varphi}{2}\right)-\left(\frac{\varphi \beta}{2}+(\beta-1)\right) \exp \left(-\frac{\varphi}{(\beta-1)}\right),
$$

which is the result found previously by Lundin and Sandahl (1978).

\section{Results}

The relativistic expressions given by Eqs. (23) and (26) for the current density and kinetic energy flux of the accelerated population for arbitrary initial thermal energy $k T$, acceleration energy $e \Phi$, and field strength ratio $\left(B / B_{o}\right)$ beneath the voltage drop, constitute the principal results derived in this paper. In this section we now evaluate these expressions for some representative situations and discuss their behaviour, derive some limiting cases, and compare the results with the non-relativistic approximations given by Eqs. (24) and (27).

In Fig. 2 we first show how the current density and kinetic energy flux vary with the field strength beneath the voltage drop, for a given temperature of the unaccelerated population and various fixed accelerating potentials. Specifically we have chosen $\mu=\left(m c^{2} / k T\right)=20$, the same value as used in Fig. 1 corresponding to an initial temperature of $\sim 25 \mathrm{keV}$ for electrons, and accelerating potentials given by $\varphi=(e \Phi / k T)=2,20$, and 200, corresponding to $\Phi$ values of $\sim 50, \sim 500$, and $\sim 5000 \mathrm{kV}$ for electrons. These accelerating potentials can therefore also be written as $\varphi / \mu=\left(e \Phi / m c^{2}\right)=0.1,1$, and 10 , thus spanning the range from the non-relativistic to the fully relativistic regimes, and corresponding to the momentum-space plots shown in Figs. 1a to 1c. Results are shown for the normalised current density and kinetic energy flux versus $\beta=\left(B / B_{o}\right)$ in the log-log plots in Figs. $2 \mathrm{a}$ and b, where the solid lines show values derived from the relativistic general expressions given by Eqs. (23) and (26), while the dotted lines correspond to the non-relativistic approximations given by Eqs. (24) and (27). From the bottom to the top of each plot, the lines shown correspond to $(e \Phi / k T)=2,20$, and 200, respectively, or equivalently to $\left(e \Phi / m c^{2}\right)=0.1,1$, and 10 . It can be seen that the relativistic values closely follow the non-relativistic approximations in the lower curves for which $(e \Phi / k T)=2$ (or equivalently $\left(e \Phi / m c^{2}\right)=0.1$ ), as expected. However, increasing deviations occur in the large $\left(B / B_{o}\right)$ regime as the potential increases, with the relativistic values being increasingly larger than those of the non-relativistic approximations.

Examining these curves in more detail, it can be seen that each displays an initial rise which is linear in $\left(B / B_{o}\right)$ (i.e. a rise with unit slope in the log-log plot), followed by an approach to a constant value as $\left(B / B_{o}\right)$ becomes large. Physically, the initial rise is due simply to conservation of particles on the magnetic flux tubes as the field strength rises and their cross-sectional area correspondingly falls. Beneath the voltage drop, the accelerated particle distributions are beamed increasingly along the field direction as the potential rises compared with the particle thermal energy perpendicular to the field (as seen in Fig. 1), such that an initial 
$\left(B / B_{o}\right)$ regime occurs in which mirroring is insignificant. In this case conservation of particles implies that $j_{\|} A \approx$ constant and $W_{\|} A \approx$ constant for the accelerated distribution, where $A$ is the cross-sectional area of the flux tube, and since also $B A \approx$ constant from magnetic flux conservation, this implies $j_{\|} / B \approx$ constant and $W_{\|} / B \approx$ constant. We can thus write $j_{\|} / B \approx j_{\|}^{\prime} / B_{o}$ and $W_{\|} / B \approx W_{\|}^{\prime} / B_{o}$, where $j_{\| \mid}^{\prime}$ and $W_{\|}^{\prime}$ correspond to the values for the accelerated population immediately beneath the voltage drop where $B=B_{o}$. The values of these latter quantities, in terms of the values for the unaccelerated population, follow directly from the requirements of conservation of particles and energy. First, it is clear that the flux of particles across the acceleration region is preserved, so that $j_{\|}^{\prime}=j_{\| o}$. Second, from Poynting's theorem, the kinetic energy flux of the particles across the acceleration region is increased by the volume integral of $j . E$ on the flux tube, so that $W_{\|}^{\prime}=W_{\| o}+j_{\| o} \Phi$. For sufficiently small $\left(B / B_{o}\right)$ we thus have

$$
\left(\frac{j_{\|}}{j_{\| o}}\right)_{\lim A}=\left(\frac{B}{B_{o}}\right),
$$

independent of the accelerating potential, and

$$
\begin{aligned}
\left(\frac{\mathcal{W}_{\|}}{\mathcal{W}_{\| o}}\right)_{\lim A} & =\left(\frac{B}{B_{o}}\right)\left(1+\frac{j_{\| o} \Phi}{\mathcal{W}_{\| o}}\right) \\
& =\left(\frac{B}{B_{o}}\right)\left(1+\frac{\left(\frac{e \Phi}{k T}\right)\left(\left(\frac{m c^{2}}{k T}\right)+1\right)}{\left(2\left(\frac{m c^{2}}{k T}\right)+3\right)}\right),
\end{aligned}
$$

where we have employed the expressions for $j_{\| o}$ and $W_{\| o}$ given in Eq. (17), and have written out the expressions in full for ease of comprehension. Mathematically, these limiting behaviours for sufficiently small $\left(B / B_{o}\right)$ follow from Eqs. (23) and (26) in the limit that

$$
\left(\varepsilon_{L}\left(\frac{\pi}{2}\right)-\varphi-\mu\right) \gg 1 \text {, }
$$

such that the terms containing the exponentials can be neglected. Equations (23) and (26) then reduce identically to Eqs. (28) and (29). Neglect of the corresponding exponential terms in the non-relativistic expressions Eqs. (24) and (27) then similarly yields

$$
\left(\frac{j_{\|}}{j_{\| o}}\right)_{\lim A} \approx\left(\frac{B}{B_{o}}\right)
$$

and

$$
\left(\frac{\mathcal{W}_{\|}}{\mathcal{W}_{\| o}}\right)_{\lim A} \approx\left(\frac{B}{B_{o}}\right)\left(1+\frac{1}{2}\left(\frac{e \Phi}{k T}\right)\right),
$$

which are the same as Eqs. (28) and (29) when $\mu \gg 1$. The physical meaning of the inequality in Eq. (30) is that the value of the accelerated distribution function given by Eq. (6) at the limiting energy at $90^{\circ}$ pitch angle, $\mathcal{E}_{L}\left(\frac{\pi}{2}\right)$, is much less than the maximum value $f_{o}$, i.e. that $f\left(\mathcal{E}_{L}\left(\frac{\pi}{2}\right)\right) / f_{o} \ll 1$, such that the flux of locally mirroring particles is negligible, in conformity with the above physical discussion. The limiting expressions given by Eqs. (28) and (29) are shown by the sloping dashed lines on the left-hand sides of Figs. 2a and b, respectively, and can be seen to correspond very closely to the full expressions shown by the solid lines for sufficiently small $\left(B / B_{o}\right)$.

At larger $\left(B / B_{o}\right)$, however, the curves in Fig. 2 are seen to asymptote to constant values which increase with the accelerating potential. This corresponds to the regime in which the field strength has become large enough that the accelerated population is rendered essentially isotropic by particle mirroring, and hence independent of $\left(B / B_{o}\right)$. This limit is reached when

$$
\left(\varepsilon_{L}\left(\frac{\pi}{2}\right)-\varphi-\mu\right) \ll 1,
$$

such that the value of $f$ at the limiting energy $\mathcal{E}_{L}\left(\frac{\pi}{2}\right)$ at $90^{\circ}$ pitch angle is close to the maximum value $f_{o}$, and hence is so at all pitch angles. The limiting values of the current density and kinetic energy flux can then be found directly from Eqs. (23) and (26) by expanding the $R H$ sides for small $1 / \beta=\left(B_{o} / B\right)$ and retaining up to terms linear in the latter parameter, or, more simply, by putting $\varepsilon_{L}(\alpha) \approx \varphi+\mu$ for all $\alpha$ directly into the integral forms Eqs. (21) and (25), and carrying through the resulting trivial pitch angle integrals. The results in either case, which we again write out in full, are

$$
\left(\frac{j_{\|}}{j_{\| o}}\right)_{\lim B}=1+\left(\frac{e \Phi}{k T}\right)+\frac{1}{2} \frac{\left(\frac{e \Phi}{k T}\right)^{2}}{\left(\left(\frac{m c^{2}}{k T}\right)+1\right)},
$$

and

$$
\left(\frac{\mathcal{W}_{\|}}{\mathcal{W}_{\| o}}\right)_{\lim B}=1+\left(\frac{e \Phi}{k T}\right)+\frac{1}{2}\left(\frac{e \Phi}{k T}\right)^{2}+\frac{1}{2} \frac{\left(\frac{e \Phi}{k T}\right)^{3}}{\left(2\left(\frac{m c^{2}}{k T}\right)+3\right)},
$$

such that in the limit $\left(B / B_{o}\right) \rightarrow \infty$ the current density and kinetic energy flux vary monotonically as simple polynomials of the accelerating potential. The corresponding nonrelativistic limits, which can be obtained for large $\left(B / B_{o}\right)$ from Eqs. (24) and (27), are

$$
\left(\frac{j_{\|}}{j_{\| o}}\right)_{\lim B} \approx 1+\left(\frac{e \Phi}{k T}\right)
$$

and

$$
\left(\frac{\mathcal{W}_{\|}}{\mathcal{W}_{\| o}}\right)_{\lim B} \approx 1+\left(\frac{e \Phi}{k T}\right)+\frac{1}{2}\left(\frac{e \Phi}{k T}\right)^{2}
$$

thus corresponding to Eqs. (33) and (34) with the last terms on the $R H$ sides eliminated, in the limit that $\left(m c^{2} / k T\right) \gg 1$. In this case the limiting current density increases linearly with the potential, and the limiting kinetic energy flux as the square of the potential, these being the well-known results obtained previously by Knight (1973) and Lundin and Sandahl (1978), respectively. In the relativistic regime, however, it can be seen that the additional term results in the current density increasing as the square of the potential, and the kinetic energy flux as the cube, such that the relativistic values increasingly exceed the non-relativistic approximations 
as the potential increases, as seen in Fig. 2. The relativistic limits given by Eqs. (33) and (34) are shown in Figs. 2a and $\mathrm{b}$, respectively, by the horizontal dashed lines on the righthand sides of the figures, where they are seen to correspond closely to the full expressions shown by the solid lines for sufficiently large $\left(B / B_{o}\right)$.

The origins of the above dependencies of the current density and kinetic energy flux of the accelerated and mirrored particles on the accelerating potential in the non-relativistic and relativistic regimes can be understood by considering the properties of the accelerated particle shell in momentum space, as depicted in Fig. 1. In the non-relativistic regime the radius of the shell in momentum space increases as the square root of the potential (assumed large compared with $(k T / e)$ ), while its thickness varies inversely with the square root of the potential. Consequently, the volume of momentum space occupied by the accelerated mirrored particles, and hence the density, increases overall as the square root of the potential. The mean speed of the particles along the field lines also increases as the square root of the potential, so the current density overall increases in direct proportion to the potential, and the kinetic energy flux as the square, as in Eq. (35). In the relativistic regime, however, the radius of the shell in momentum space increases in direct proportion to the potential, while its thickness tends to the constant value $\sim(k T / c)$. The density of the accelerated mirrored particles in this case thus increases as the square of the potential. The mean speed of the particles along the field lines also tends to the constant value $(c / 2)$, so that overall, the current density increases as the square of the potential, and the kinetic energy flux as the cube, as in Eqs. (33) and (34).

Summarising, then, the results shown in Fig. 2, we note that the current density and the kinetic energy flux follow the limiting forms given by Eqs. (28) and (29) for sufficiently small $\left(B / B_{O}\right)$ (limit "A"), while following the limiting forms given by Eqs. (33) and (34) for sufficiently large $\left(B / B_{O}\right)$ (limit "B"). The former limit applies when the inequality in Eq. (30) is satisfied, while the latter applies when the opposite inequality in Eq. (32) is satisfied. It thus seems appropriate to take the condition which separates the two regimes to be given by

$$
\left(\varepsilon_{L}\left(\frac{\pi}{2}\right)-\varphi-\mu\right)=1,
$$

such that the distribution function given by Eq. (6) at the limiting energy at $90^{\circ}$ pitch angle is given by $f\left(\mathcal{E}_{L}\left(\frac{\pi}{2}\right)\right) / f_{o}=1 / e$. Then introducing the expression for $\mathcal{E}_{L}\left(\frac{\pi}{2}\right)$ given by Eq. (7c) and solving for $\left(B / B_{o}\right)$ for given $\varphi=(e \Phi / k T)$, yields the limiting value

$$
\left(\frac{B}{B_{o}}\right)_{\lim A-B}=1+\left(\frac{e \Phi}{k T}\right) \frac{\left(\left(\frac{e \Phi}{k T}\right)+2\left(\frac{m c^{2}}{k T}\right)+2\right)}{\left(2\left(\frac{m c^{2}}{k T}\right)+1\right)} .
$$

The limits given by Eqs. (28) and (29) then apply when $\left(B / B_{o}\right) \ll\left(B / B_{o}\right)_{\lim A-B}$, while the opposite limits given by Eqs. (33) and (34) apply when
$\left(B / B_{o}\right) \gg\left(B / B_{o}\right)_{\lim A-B}$. The limiting values of $\left(B / B_{o}\right)$ given by Eq. (37) are marked by the solid dots on the solid curves in Fig. 2, showing that they do indeed mark the transition between the two regimes of behaviour. The transition between the limiting values shown by the dashed lines in the figure has also been taken to occur at $\left(B / B_{O}\right)_{\lim A-B}$ for each accelerating potential, the resulting lines showing that the limiting value of $\left(B / B_{o}\right)$ given by Eq. (37) occurs essentially at the point of intersection between the corresponding dashed lines, as may be expected. For the lower curves in Fig. 2 corresponding to $(e \Phi / k T)=2$, the transition occurs near $\left(B / B_{o}\right) \sim 3$, consistent with the momentum-space limiting lines and distribution function contours shown in Fig. 1a. For the upper curves in Fig. 2 with $(e \Phi / k T)=20$ and 200, however, the transitions occur at increasingly large values of $\left(B / B_{o}\right)$, near $\sim 30$ and $\sim 1000$, respectively, consistent with Figs. $1 \mathrm{~b}$ and $\mathrm{c}$. Although values $\left(B / B_{o}\right) \sim 1000$ may seem rather large, we note that for the dipole field of a planetary body it simply represents the ratio between the field strength near the surface and that at a point at a distance of $\sim 10$ planetary radii, which for one of the giant planets represents a point well inside the magnetospheric cavity.

Further insight into the physical significance of these results may be obtained by plotting the current density and kinetic energy flux versus the accelerating potential at fixed $\left(B / B_{o}\right)$, as shown in Fig. 3. As in Figs. 1 and 2, we have chosen $\left(m c^{2} / k T\right)=20$, corresponding to a nonrelativistic unaccelerated population with an initial temperature of $\sim 25 \mathrm{keV}$ for electrons, and show the variations of the normalized fluxes versus $(e \Phi / k T)$ over the range from 0.1 to $10^{4}$, corresponding to potentials from $\sim 2.5 \mathrm{kV}$ to $250 \mathrm{MV}$. This range thus spans the non-relativistic and fully relativistic regimes, with $\left(e \Phi / m c^{2}\right)=(e \Phi / k T) / 20$ varying between 0.005 and 500 . The transition between these regimes (for an initially non-relativistic population), corresponding to $\left(e \Phi / m c^{2}\right)=1$, thus occurs at $(e \Phi / k T)=20$, near the middle of each plot. The lower, middle, and upper solid lines in each panel then show the relativistic values of the normalised current density and kinetic energy flux for $\left(B / B_{o}\right)=10,100$, and 1000 , respectively, obtained from Eqs. (23) and (26), while the dotted lines show the corresponding non-relativistic approximations given by Eqs. (24) and (27). The long-dashed lines also show the limiting values for $\left(B / B_{o}\right) \rightarrow \infty$ given by Eqs. (33) and (34), while the short-dashed lines show the non-relativistic approximations given by Eqs. (35a, b).

We first consider the results for the current density shown in Fig. 3a. For small accelerating potentials on the lefthand side of the figure, the value of $\left(B / B_{o}\right)$ is sufficient in each case to result in near-isotropy in the accelerated population beneath the voltage drop (i.e. the inequality in Eq. (32) is satisfied), such that the curves closely approximate the $\left(B / B_{o}\right) \rightarrow \infty$ limit given by Eq. (33) (limit "B"), shown by the long-dashed line. For $(e \Phi / k T) \gg 1$ the current density thus increases linearly with the potential in the non-relativistic regime, and as the square of the 
(a)

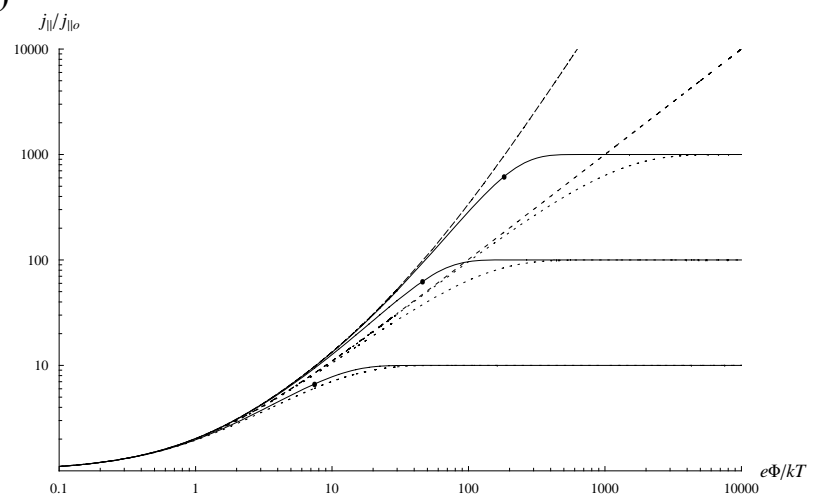

(b)

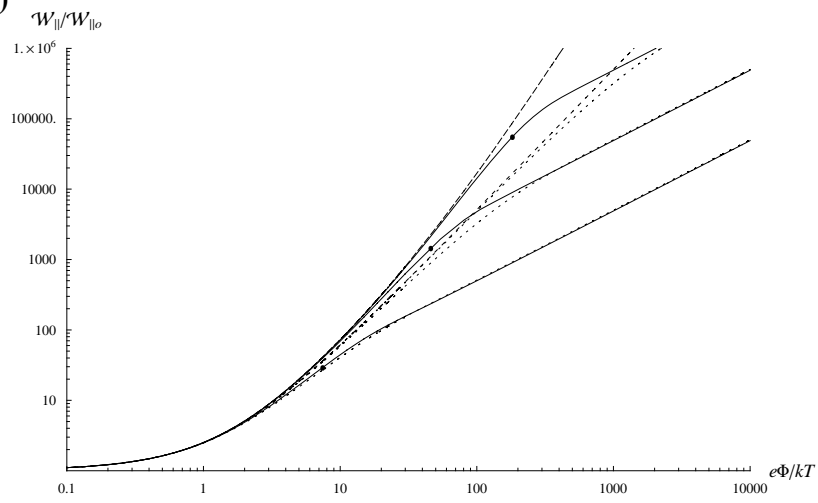

Fig. 3. Plots showing the variation of (a) the normalised field-aligned current density $\left(j_{\|} / j_{\| o}\right)$ (or number flux), and (b) the normalised field-aligned kinetic energy flux $\left(\mathcal{W}_{\|} / \mathcal{W}_{\| o}\right)$ of the accelerated population, plotted versus normalised acceleration potential $(e \Phi / k T)$ for fixed values of the magnetic field ratio $\left(B / B_{O}\right)$ beneath the voltage drop. The fluxes are normalised to the corresponding values for the unaccelerated population in each case, given by Eq. (17). The three pairs of solid and dotted lines in each plot correspond to the relativistic values and non-relativistic approximations, respectively, for $\left(B / B_{O}\right)=10,100$, and 1000, from the bottom to the top of each plot. These curves are given by Eqs. (23) and (24) for the current density, and Eqs. (26) and (27) for the kinetic energy flux. As in previous figures, all curves are for a non-relativistic unaccelerated population satisfying $\left(m c^{2} / k T\right)=20$, corresponding to $k T \sim 25 \mathrm{keV}$ for electrons. Relativistic accelerations satisfying $\left(e \Phi / m c^{2}\right) \geq 1$ thus correspond to $(e \Phi / k T) \geq 20$. The long- and medium-dashed lines correspond to the relativistic value and the non-relativistic approximation, respectively, of the limiting fluxes for $\left(B / B_{o}\right) \rightarrow \infty$ (limit "B"), given by Eqs. (33) and (35a) for the current density, and Eqs. (34) and (35b) for the kinetic energy flux. The solution curves in each case follow this limiting behaviour for sufficiently small $(e \Phi / k T)$, before asymptoting to the opposite limit (limit "A") given by Eqs. (28) and (29) at sufficiently large ( $e \Phi / k T)$. The transition between these two limiting regimes on each solid curve is shown by the solid dot, whose position is determined by the condition given by Eq. (36), specifically by Eq. (38).

potential in the relativistic regime. Eventually, however, as $(e \Phi / k T)$ increases, the given value of $\left(B / B_{o}\right)$ becomes insufficient to maintain isotropy, so that a transition takes place to the opposite limit (limit "A") in which local mirroring is insignificant, such that $\left(j_{\| o} / j_{\|}\right) \approx\left(B / B_{o}\right)$, as given by Eq. (28). Each solid curve thus eventually ceases to increase with $(e \Phi / k T)$, and asymptotes to its corresponding limiting value of $\left(j_{\|} / j_{\| o}\right)_{\lim A}=\left(B / B_{o}\right)$. The dotted curves showing the non-relativistic behaviour asymptote to the same values even in the fully relativistic regime, since this limiting value is determined only by conservation of the particle number flux. The transition between the two regimes occurs near the point where Eq. (36) is satisfied, yielding a quadratic equation for $(e \Phi / k T)_{\lim A-B}$ at fixed $\left(B / B_{o}\right)$ which is equivalent to Eq. (37), given by

$$
\begin{aligned}
& \left(\frac{e \Phi}{k T}\right)_{\lim A-B}= \\
& \sqrt{\left(\left(\frac{m c^{2}}{k T}\right)+1\right)^{2}+\left(\frac{B}{B_{o}}-1\right)\left(2\left(\frac{m c^{2}}{k T}\right)+1\right)} \\
& -\left(\left(\frac{m c^{2}}{k T}\right)+1\right) .
\end{aligned}
$$

As in Fig. 2, this point is shown by the solid dots on each solid line, and marks the transition between the two regimes of behaviour in each case.
Turning now to the curves for the kinetic energy flux shown in Fig. 3b, it can be seen that their behaviour follows expectations based on the above discussion. For sufficiently small $(e \Phi / k T)$ the curves follow the $\left(B / B_{o}\right) \rightarrow \infty$ limiting behaviour (limit "B") for a near-isotropic accelerated population given by Eq. (34), increasing (when $(e \Phi / k T) \gg 1$ ) as the square of the potential in the non-relativistic regime, and as the cube of the potential in the relativistic regime. However, in the vicinity of the potential shown by the solid dot on each curve, given again by Eq. (38), a transition takes place to the opposite regime of behaviour (limit "A") for sufficiently large $(e \Phi / k T)$, given by Eq. (29), determined by conservation of kinetic energy flux along the magnetic flux tubes beneath the voltage drop. When $\left(m c^{2} / k T\right) \gg 1$ and $(e \Phi / k T) \gg 1$, Eq. (29) becomes approximately $\left(\mathcal{W}_{\|} / W_{\| o}\right) \approx \frac{1}{2}\left(B / B_{o}\right)(e \Phi / k T)$, such that the kinetic energy flux is proportional to the field ratio $\left(B / B_{o}\right)$ and increases linearly with the potential, as seen on the $R H$ side of Fig. 3b.

\section{Implications for auroral voltages}

Let us now consider explicitly the implications of the results shown in Fig. 3 for the flow of electric current along the magnetic field lines between some central planetary body and its external magnetosphere. Suppose that the magnetosphereionosphere coupling current circuit requires the flow of an 
upward-directed current of magnitude $j_{||}$just above the ionosphere, where the field strength is $B$, to be carried by magnetospheric electrons, and that this is larger than the maximum current that the latter unaccelerated population can carry, $j_{\| o}$, depending on the magnetospheric number density $N_{o}$ and the temperature $T$ through Eq. (17a). The range of solutions of the normalised accelerating potential $(e \Phi / k T)$ and acceleration region height (or equivalently the field ratio $\left.\left(B / B_{o}\right)\right)$ that will produce the required current density at the required field strength in the ionosphere is then determined by the solution curves such as those shown in Fig. 3a (for $\left(m c^{2} / k T\right)=20$ in that case) which pass through the horizontal line representing the required value of the current ratio $\left(j_{\|} / j_{\| o}\right)$. Consideration of the form of the curves in Fig. 3a then shows that the range of potentials varies from a minimum value given by the solution to Eq. (33), upwards to infinity, with a range of corresponding $\left(B / B_{o}\right)$ values varying downwards from infinity to $\left(B / B_{o}\right)=\left(j_{\|} / j_{\| o}\right)$.

Paraphrasing this discussion, therefore, in order to carry a current $\left(j_{\|} / j_{\| o}\right)>1$, the minimum height of the acceleration region is given by the condition $\left(B / B_{o}\right)=\left(j_{\|} / j_{\| \mid}\right)$, corresponding to an infinite accelerating potential, such that the total flux of down-going electrons at that altitude is accelerated into the ionosphere to produce current density $j_{\| \mid}$at field strength $B$. Assuming a dipole magnetic field in which the field strength falls along the polar field lines approximately as the cube of the radial distance from the planetary centre, the minimum radial distance of the acceleration region $R_{o}$ is then given by

$$
\left(\frac{R_{o}}{R}\right)_{\min } \approx\left(\frac{j_{\|}}{j_{\| o}}\right)^{1 / 3}
$$

where $R$ is the radial distance of the ionosphere, usually essentially equal to the radius of the body concerned. This is the same limit as employed in previous studies e.g. by Cowley et al. (2003, 2004, 2005), since it depends only on particle flux conservation and is independent of relativistic considerations. For acceleration regions located increasingly above this minimum height, however, the required potential falls to a minimum value as the field strength $B_{o}$ falls to low values (strictly to zero), determined by the solution of the quadratic expression in Eq. (33). The corresponding relativistic expression for the minimum value of the particle kinetic energy flux is then given by Eq. (34).

It is important to note, however, that the acceleration region does not generally have to be located far above the minimum height for the required potential to fall to values comparable with the lower limit given by Eq. (33). Examples are given in Fig. 4, where we show the variation of the normalised accelerating potential $(e \Phi / k T)$ versus the field ratio $\left(B / B_{o}\right)$ required to produce fixed normalised current densities, for $\left(m c^{2} / k T\right)=20$ (thus corresponding to Fig. $3 \mathrm{a}$ and other figures). From bottom to top in the figure, the solid lines show results for $\left(j_{\|} / j_{\| o}\right)=50,100$, and 200. For each curve the corresponding horizontal dashed line shows the limiting minimum potential for $\left(B / B_{o}\right) \rightarrow \infty$

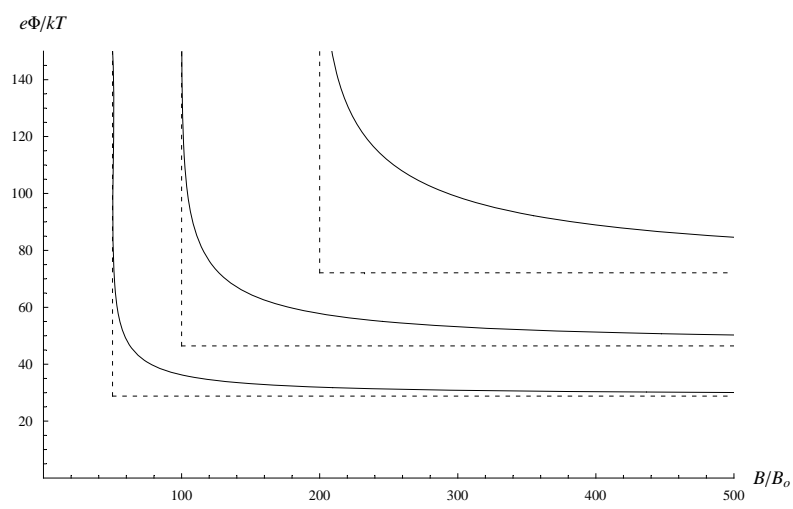

Fig. 4. Plot showing the normalized accelerating potential $(e \Phi / k T)$ required to produce a fixed normalised field-aligned current density $\left(j_{\|} / j_{\| o}\right)$, versus the field ratio $\left(B / B_{O}\right)$, for a fixed unaccelerated population with temperature given by $\left(m c^{2} / k T\right)=20$ (as in Figs. 1-3). From bottom to top the three solid lines are for $\left(j_{\|} / j_{\| o}\right)=50,100$, and 200 , respectively. For each curve, the corresponding horizontal dashed line shows the minimum accelerating potential given by the solution to Eq. (33), which applies in the limit $\left(B / B_{O}\right) \rightarrow \infty$ (limit "B"). The vertical dashed lines show the minimum possible value of $\left(B / B_{o}\right)$ (equal to $\left(j_{\|} / j_{\| o}\right)$ in each case), where the required potential diverges to infinity (limit "A"). It can be seen that the required voltages drop rapidly toward the minimum value for $\left(B / B_{O}\right)$ values only modestly larger than the minimum, by factors of two or three.

given by the solution to Eq. (33). Each corresponding vertical dashed line also marks the minimum possible value of $\left(B / B_{o}\right)$ for that normalised current density (just given by $\left.\left(B / B_{o}\right)=\left(j_{\|} / j_{\| o}\right)\right)$, corresponding to the minimum height where the required acceleration potential diverges to infinity. It can be seen that the required acceleration potential falls rapidly for larger $\left(B / B_{O}\right)$ values towards the minimum value given by Eq. (33), and is close to the latter value for field strength ratios that are factors of only two or three larger than the minimum value. Consequently, it seems appropriate to take the minimum value as a reasonable measure of the acceleration potential that will form in practice for a given field-aligned current, given by Eq. (33), as has been routinely assumed in previous work using the non-relativistic approximations. The corresponding minimum value of the precipitating kinetic energy flux is then that given by Eq. (34).

In Figs. 5a and $\mathrm{b}$ we thus finally show the variations of the field-aligned current density and kinetic energy flux, respectively, normalised to the corresponding values for the unaccelerated population, plotted versus the minimum acceleration potential energy over the range from $1 \mathrm{keV}$ to $100 \mathrm{MeV}$, as given by Eqs. (33) and (34). The upper, middle, and lower solid lines in each figure show the flux values given by these equations for thermal energies of the unaccelerated population given by $k T=1,10$, and $100 \mathrm{keV}$, respectively. The dotted lines show the corresponding values for the non-relativistic approximations given by Eqs. (35a, b). It can be seen that the solid lines follow these approximations in the non-relativistic regime for $e \Phi<m c^{2} \approx 511 \mathrm{keV}$, the current density increasing 
(a)

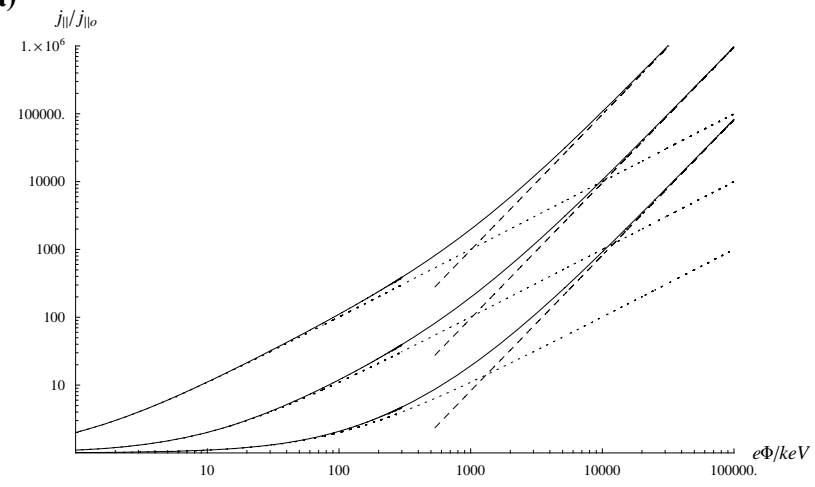

(b)

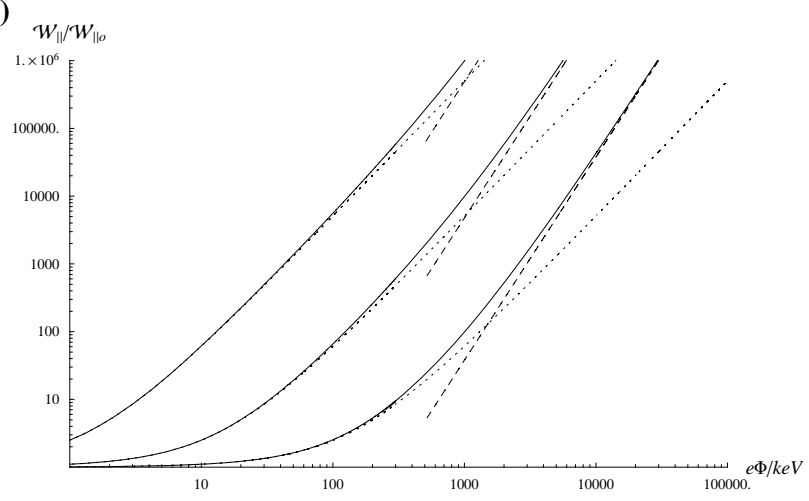

Fig. 5. Plots showing the variation of (a) the normalised field-aligned current density $\left(j_{\|} / j_{\|}\right)$(or number flux), and (b) the normalized field-aligned kinetic energy flux $\left(\mathcal{W}_{\|} / \mathcal{W}_{\| o}\right)$, versus the minimum acceleration potential energy $e \Phi$ in keV, given by Eqs. (33) and (34), respectively. The fluxes are normalised to the corresponding values for the unaccelerated population in each case, given by Eq. (17). The upper, middle, and lower solid lines in each panel show the relativistic flux values for $k T=1,10$, and $100 \mathrm{keV}$, respectively, while the nonrelativistic approximations given by Eqs. (35a, b) are shown by the corresponding dotted lines. The long-dashed lines show the final term only on the $R H$ sides of Eqs. (33) and (34), plotted in the relativistic region where $e \Phi \geq m c^{2} \approx 511 \mathrm{keV}$.

linearly with the potential, and the kinetic energy flux as the square, in the regime where $e \Phi>k T$ for each curve. However, the relativistic flux values increasingly diverge to larger values in the relativistic regime where $e \Phi>m c^{2}$. In this regime the normalised flux values are dominated by the final term on the $R H$ sides of Eqs. (33) and (34), as indicated by the long-dashed lines in Fig. 5, which show the final terms alone for acceleration potential energies above $m c^{2} \approx 511 \mathrm{keV}$. Under these relativistic conditions we thus find that the current density increases as the square of the potential, or equivalently that the potential increases as the square root of the current density, and that the kinetic energy flux increases as the cube of the potential, or equivalently as the current density to the three-halves power.

\section{Summary}

Large-scale current systems flowing between the magnetospheres and ionospheres of planetary bodies are set up whenever they exchange momentum via the magnetic field that links them. The forces are exerted via the cross-field currents flowing in these bodies of plasma, while the current circuit is completed by upward- and downward-directed currents flowing along the linking field lines. The upward-directed field-aligned currents are of special significance, since they are generally dominantly carried by downward-precipitating electrons from the hot, tenuous magnetospheric plasma. The current that such particles can carry is, however, subject to strict limitation, and when the requirement for current by the circuit exceeds this limit, field-aligned voltages must appear along the field lines which accelerate the magnetospheric electrons into the ionosphere, thereby increasing both the current (number flux) and the kinetic energy flux of the precipitating particles. Knight (1973) originally calculated the current-voltage relationship for hot precipitating electrons in the non-relativistic limit, assuming an isotropic Maxwellian source population, and found that for an acceleration region at sufficient altitude (for which the required accelerating potential is a minimum), the current (assumed well above the limiting value) increases linearly with the voltage. Lundin and Sandahl (1978) subsequently calculated the precipitating energy flux on the same basis, and found that it increases as the square of the potential, or equivalently as the square of the current density. These results have subsequently been extended by a number of authors to include other forms for the source distribution, and have been applied extensively to the auroral acceleration process in the terrestrial system.

The non-relativistic approximation is indeed well satisfied in the terrestrial system, where accelerating potentials are typically in the range $\sim 1-10 \mathrm{kV}$, such that the energy of the accelerated particles is much less than the electron rest mass of $\sim 511 \mathrm{keV}$. Indeed, the total voltage associated with the solar wind interaction across the whole magnetosphere, representing the maximum possible potential in the static case, is only $\sim 50-100 \mathrm{kV}$. However, observations of the "main oval" auroras in the jovian system indicate that electrons are routinely accelerated along the field to energies of $\sim 100 \mathrm{keV}$, sometimes reaching several hundred $\mathrm{keV}$, these values comparing with cross-field voltages in the outer and middle magnetosphere of order $\sim 10 \mathrm{MV}$. Such observations thus suggest the need to consider the relativistic regime in this case. Related processes in astrophysical systems have also been discussed in which electrons are accelerated to highly relativistic energies.

In this paper we have therefore considered the relativistic problem of the field-aligned acceleration of auroral electrons through an arbitrary field-aligned potential, assuming for simplicity, and in keeping with Knight (1973) and many previous works, that the source population is an isotropic Maxwellian, of arbitrary temperature. We have mapped the 
particle distribution function across the voltage drop into the region of increasing magnetic field strength on the other side using Liouville's theorem, together with conservation of total energy and the magnetic moment invariant, assuming for simplicity that the acceleration region is sufficiently compact along the field that magnetic mirroring within it can be neglected. By integration of suitable moments over the accelerated particle distribution, we have then obtained exact analytic expressions for the field-aligned current density (number flux) and kinetic energy flux of the accelerated particles (Eqs. 23 and 26), as functions of the temperature and density of the source plasma, the accelerating potential, and the field strength beneath the acceleration region. We have also shown that these expressions reduce to the well-known non-relativistic results of Knight (1973) and Lundin and Sandahl (1978) in the appropriate limit (Eqs. 24 and 27).

We have then examined the nature of the solutions, which, as in the non-relativistic case, show two limiting behaviours with a transition between them. The first limit occurs for sufficiently small magnetic field strength relative to that in the acceleration region for a fixed accelerating potential (or equivalently for a sufficiently high accelerating potential relative to the initial temperature for a fixed field strength), such that particle mirroring from the accelerated population is essentially negligible. In this case the current density and kinetic energy flux are simply governed by conservation of particles and energy, increasing linearly with the magnetic field strength (Eqs. 28 and 29). The second limit then occurs for sufficiently large magnetic field strength relative to that in the acceleration region for a fixed accelerating potential (or equivalently for a sufficiently small accelerating potential relative to the initial temperature for a fixed field strength), such that the accelerated population becomes fully isotropic due to magnetic mirroring. In this case the current density and the kinetic energy flux become independent of the magnetic field strength, and instead vary as simple polynomials of the accelerating potential, a quadratic for the current density, and a cubic for the kinetic energy flux (Eqs. 33 and 34). The condition which separates these two limiting behaviours has also been obtained, given by Eq. (37) for the limiting field ratio for a given accelerating potential (or equivalently Eq. 38 for the limiting accelerating potential for a given field ratio).

We have also briefly discussed the implications of these results for auroral electron acceleration in regions of upwarddirected field-aligned current. For any given value of the field-aligned current density at the top of the ionosphere, larger than the maximum value for the unaccelerated population, a range of possible solutions exist, depending on the height of the acceleration region above the ionosphere (and hence the field strength relative to the ionospheric value), and the consequent accelerating potential required. However, the first of the above limits (limit "A") requires from number flux conservation that the acceleration region should lie above a minimum distance (given approximately by Eq. 39 for a dipole field), at which the required accelerating potential becomes infinite. Above this height, however, the required ac- celerating potential falls rapidly to a minimum value given by the second of the above limits (limit "B"), which can thus be taken as a reasonable estimate of the potential likely to occur in practice. When the required current density considerably exceeds the maximum for the unaccelerated population, the current density depends linearly on the accelerating potential in the non-relativistic regime in this limit, and the kinetic energy flux as the square, as found previously by Knight (1973) and Lundin and Sandahl (1978). However, when the accelerating potential energy exceeds the electron rest energy in the relativistic regime, we find that the current density instead increases as the square of the potential, or in other words that the potential increases as the square root of the required current. In the same limit, the kinetic energy flux increases as the cube of the potential, or equivalently as the three-halves power of the current density.

Acknowledgements. This research was supported by PPARC grant $\mathrm{PPA} / \mathrm{G} / \mathrm{O} / 2003 / 00013$. It is dedicated as a contribution to Einstein Year 2005.

Topical Editor T. Pulkkinen thanks L. Lyons and P. Janhunen for their help in evaluating this paper.

\section{References}

Antonova, E. E. and Tverskoy, B.A.: Nature of the electron precipitation band of the 'inverted V' type and of the Harang discontinuity in the evening sector of the auroral ionosphere, Geomagn. Aeronomy (English translation), 15, 85-92, 1975.

Begelman, M. C., Ergun, R. E., and Rees, M. J.: Cyclotron maser emission from blazar jets?, Ap. J., 625, 51-59, 2005.

Bosqued, J. M., Maurel, C., Sauvaud, J. A., Kovrazhkin, R. A., and Galperin, Y. I.: Observations of auroral electron invertedV structures by the AUREOL-3 satellite, Planet. Space Sci., 34, 255-269, 1986.

Carlson, H. C. and Cowley, S. W. H.: Accelerated polar rain electrons as the source of sun-aligned arcs in the polar cap during northward interplanetary magnetic field conditions, J. Geophys. Res., 110, A05302, doi:10.1029/2004JA010669, 2005.

Clemmow, P. C. and Dougherty, J. P.: Electrodynamics of particles and plasmas, Addison-Wesley Publ. Co., 1969.

Cowley, S. W. H.: Magnetosphere-ionosphere interactions: A tutorial review, in: Magnetospheric Current Systems, edited by: Ohtani, S., Fujii, R., Hesse, M., and Lysak, R. L., Geophys. Monograph, 118, AGU Publ., Washington, USA, 91-106, 2000.

Cowley, S. W. H. and Bunce, E. J.: Origin of the main auroral oval in Jupiter's coupled magnetosphere-ionosphere system, Planet. Space Sci., 49, 1067-1088, 2001.

Cowley, S. W. H., Bunce, E. J., and Nichols, J. D.: Origins of Jupiter's main oval auroral emissions, J. Geophys. Res., 108(A4), 8002, doi:10.1029/2002JA009329, 2003.

Cowley, S. W. H., Bunce, E. J., and O'Rourke, J. M.: A simple quantitative model of plasma flows and currents in Saturn's polar ionosphere, J. Geophys. Res., 109, A05212, doi:10.1029/2003JA010375, 2004.

Cowley, S. W. H., Alexeev, I. I., Belenkaya, E. S., Bunce, E. J., Cottis, C. E., Kalegaev, V. V., Nichols, J. D., Prangé, R., and Wilson, F. J.: A simple axi-symmetric model of magnetosphereionosphere coupling currents in Jupiter's polar ionosphere, J. Geophys. Res., 110, A11209, doi:10.1029/2005JA011237, 2005. 
Dors, E. E. and Kletzing, C. A.: Effects of suprathermal tails on auroral electrodynamics, J. Geophys. Res., 104, 6783-6796, 1999.

Ergun, R. E., Carlson, C. W., McFadden, J. P., Delory, G. T., Strangeway, R. J., and Pritchett, P. L.: Electron-cyclotron maser driven by charged particle acceleration from magnetic fieldaligned electric fields, Ap. J., 538, 456-466, 2000.

Fridman, M. and Lemaire, J.: Relationship between auroral electron fluxes and field aligned electric potential differences, J. Geophys. Res., 85, 664-670, 1980.

Gustin, J., Gérard, J.-C., Grodent, D., Cowley, S. W. H., Clarke, J. T., and Grard, A.: Energy-flux relationship in the FUV jovian aurora deduced from HST-STIS spectral observations, J. Geophys. Res., 109, A10205, doi:10.1029/2003JA010365, 2004.

Haerendel, G.: Auroral acceleration in astrophysical plasmas, Phys. Plasmas, 8, 2365-2370, 2001.

Janhunen, P. and Olsson, A.: The current-voltage relation revisited: Exact and approximate formulas with almost general validity for hot magnetospheric electrons for bi-Maxwellian and kappa distributions, Ann. Geophys., 16, 292-297, 1998,

\section{SRef-ID: 1432-0576/ag/1998-16-292.}

Knight, S.: Parallel electric fields, Planet. Space Sci., 21, 741-750, 1973.

Korth, H., Anderson, B. J., Frey, H. U., Immel, T. J., and Mende, S. B.: Conditions governing localized highlatitude dayside aurora, Geophys. Res. Lett., 31(4), L04806, doi:10.1029/2003GL018911, 2004.

Lu, G., Reiff, P. H., Burch, J. L., and Winningham, J. D.: On the auroral current-voltage relationship, J. Geophys. Res., 96, 35233531, 1991

Lundin, R. and Sandahl, I.: Some characteristics of the parallel electric field acceleration of electrons over discrete auroral arcs as observed from two rocket flights, Symposium on European Rocket Research, ESA SP-135, p. 125, 1978.

Lyons, L. R.: Generation of large-scale regions of auroral currents, electric potentials, and precipitation by the divergence of the convection electric field, J. Geophys. Res., 85, 17-24, 1980.
Lyons, L. R.: Discrete aurora as the direct result of an inferred, high-altitude generating potential distribution, J. Geophys. Res., $86,1-8,1981$.

Nichols, J. D. and Cowley, S. W. H.: Magnetosphere-ionosphere coupling currents in Jupiter's middle magnetosphere: Effect of precipitation-induced enhancement of the ionospheric Pedersen conductivity, Ann. Geophys., 22, 1799-1827, 2004,

SRef-ID: 1432-0576/ag/2004-22-1799.

Nichols, J. D. and Cowley, S. W. H.: Magnetosphere-ionosphere coupling currents in Jupiter's middle magnetosphere: Effect of magnetosphere-ionosphere decoupling by field-aligned auroral voltages, Ann. Geophys., 23, 799-808, 2005, SRef-ID: 1432-0576/ag/2005-23-799.

Olsson, A., Eriksson, A. I., and Janhunen, P.: On the current-voltage relationship in auroral breakups and westwards-travelling surges, Ann. Geophys., 14, 1265-1273, 1996,

SRef-ID: 1432-0576/ag/1996-14-1265.

Olsson, A., Andersson, L., Eriksson, A. I., Clemmons, J., Erlandsson, R. E., Reeves, G., Hughes, T., and Murphree, J. S.: Freja studies of the current-voltage relation in substorm-related events, J. Geophys. Res., 103, 4285-4301, 1998.

Paschmann, G., Haaland S., and Treumann, R. (Eds.): Auroral plasma physics, Space Sci. Rev., vol. 103, 2002.

Pierrard, V.: New model of magnetospheric current-voltage relationship, J. Geophys. Res., 101, 2669-2675, 1996.

Schriver, D., Asour-Abdalla, M., Strangeway, R. J., Richard, R. L., Klezting, C., Dotan, Y., and Wygant, J.: FAST/Polar conjunction study of field-aligned auroral acceleration and corresponding magnetotail drivers, J. Geophys. Res., 108(A9), 8020, doi:10.1029/2002JA009426, 2003.

Shiokawa, K., Fukunishi, H., Yamagishi, H., Miyaoka, H., Fujii, R., and Tohyama, F.: Rocket observations of the magnetosphereionosphere coupling processes in quiet and active arcs, J. Geophys. Res., 95, 10 679-10 686, 1990 